\title{
Relation between Uric Acid and Alzheimer's Disease in Elderly Jordanians
}

\author{
Eman Al-khateeb ${ }^{\mathrm{a}, *}$, Arwa Althaher $^{\mathrm{a}}$, Mohammed Al-khateeb $^{\mathrm{b}}$, Hanan Al-Musawi $^{\mathrm{c}}$, Ola Azzouqah ${ }^{\mathrm{c}}$, \\ Samir Al-Shweikic and Yanal Shafagoj ${ }^{\mathrm{a}}$ \\ ${ }^{a}$ Department of Physiology \& Biochemistry, Faculty of Medicine, University of Jordan, Amman, Jordan \\ ${ }^{\mathrm{b}}$ Department of Pathology, Faculty of Medicine, University of Jordan, Amman, Jordan \\ ${ }^{\mathrm{c}}$ Medical student, Faculty of Medicine, University of Jordan, Amman, Jordan
}

Accepted 29 September 2014

\begin{abstract}
.
Background: It has been suggested that oxidative injuries have a role in the pathogenesis of Alzheimer's disease (AD). Uric acid (UA) has a contradictory effect on cognitive function and several lines of evidence suggest that UA may modulate outcome in neurological diseases.

Objectives: Many studies investigated serum UA levels in AD patients, but to date, results from these observational studies are conflicting. In this study, we assess whether serum UA levels would be altered in the AD Jordanian patients compared to those of the healthy controls.

Methods: Serum UA and lipid profile levels were measured in 41 AD patients and 40 healthy controls. Results were statistically evaluated at $p<0.05$ level of significance. The Arabic version of the Mini-Mental State Examination (MMSE-A) was used to evaluate the cognitive functions of all participants.

Results: Demographic variables indicate that individuals that are illiterate demonstrate a 7.5 fold $(p=0.033)$ increase in risk of developing AD. The AD group shows 12.6\% lower serum UA level than control subjects and the difference between groups is statistically significant $(p=0.033)$. No significant differences could be found between the two groups in lipid profile levels. Pearson correlation coefficients and Multivarient linear regression show no significant correlation between MMSE and continuous variables in $\mathrm{AD}$ patients except for age.

Conclusion: The results suggest that serum UA levels are significantly lower in AD patients in comparison to control subjects. UA may have a protective role against $\mathrm{AD}$; however this role needs further investigations.
\end{abstract}

Keywords: Aging, Alzheimer's disease, biomarkers, cholesterol, dementia, lipid profile, uric acid

\section{INTRODUCTION}

According to the UN (2011) 5.5\% of Jordan's current 6.25 million inhabitants are over the age of 60 , and this is expected to increase dramatically by 2050 . We estimate that there are currently 16,500 people with clinically significant Alzheimer's disease (AD)

${ }^{*}$ Correspondence to: Eman Al-khateeb, MBChB, MSc, $\mathrm{PhD}$, Department of Physiology and Biochemistry, Faculty of Medicine, University of Jordan, P.O. Box 13490, Amman 11942, Jordan. Tel.: +962 796596390/ +962 65355000 23479; Fax: +962 65300820 E-mails: profemanalkhateeb@yahoo.com; e.alkhateeb@ju.edu.jo. and this will increase over the next forty years [1]. AD is the most common neurodegenerative dysfunction of the central nervous system and has been associated with oxidative stress and vascular pathology $[2,3]$. Cerebral tissue seems to be particularly vulnerable to oxidative damage because of its high oxygen consumption, low content of antioxidants, and high content of polyunsaturated fatty acids of neuronal membranes [4].

Cholesterol plays central role in promoting the production of amyloid- $\beta$, the main component of the amyloid plaques found in the brains of $\mathrm{AD}$ patients, and possibly the progression of $\mathrm{AD} \mathrm{[5].} \mathrm{The} \mathrm{role} \mathrm{of} \mathrm{elevated}$ 
cholesterol in dementia remains unclear. The Framingham study demonstrated that baseline and long-term average serum total cholesterol levels are not associated with the risk for incident AD [6]. Furthermore, some investigators found no difference in plasma high density lipoprotein (HDL) and low density lipoprotein (LDL) levels between AD and controls [7].

Uric acid (UA) is the final product of purine metabolism and its measurement used in the diagnosis of numerous renal and metabolic disorders. It is associated with an increased risk for myocardial infarction, stroke, and cardiovascular mortality $[8,9]$. It has been hypothesized that UA may stimulate vascular smooth muscle cell proliferation either via the inflammatory properties of the soluble UA itself or via its effect on the endothelial function by impairing nitric oxide production [8, 10]; on the other hand, UA is considered as a major natural antioxidant in plasma that reduce oxidative stress and protect against free radicals [11]. Therefore the different properties of UA might have contradictory effects on the risk of dementia and cognitive function.

It is not clear whether UA is important to the development or progression of AD [12-14]. Cross-sectional studies of serum UA have identified lower concentration in $\mathrm{AD}$ and mild cognitive impairment (MCI) patients compared to healthy controls [12, 13], but another report found no difference [15]. A secondary analysis of MCI clinical trial data found that among placebo treated subjects, lower serum UA was associated with more rapid cognitive decline and higher incidence of AD over 3 years [14].

Among possible markers of age-related cognitive decline, UA is controversial because it has antioxidant properties but is increased in diseases that often lead to cognitive impairment. Many researchers including our group have recently focused on identifying biomarkers of dementia and AD [16]. Such markers may play a key role in the early diagnosis and management of dementia. Measuring serum UA is affordable, accurate, and minimally invasive thus representing an appealing biological marker for AD.

In this study, we analyzed the relationship between serum UA and cognitive functioning in a sample of reasonably healthy elderly adults and compared them to AD patients. We focused on those with serum UA levels in the normal range because finding an association between cognitive functioning and serum UA levels in this range would have significant implications for both public health and treatment. We hypothesize that mildly elevated but normal levels of serum UA may protect against dementia and $\mathrm{AD}$.

\section{SUBJECTS AND METHODS}

\section{Subjects}

The sample population for this cross-sectional study is patients in the senior homes and Jordan University Hospital with a diagnosis of probable AD. Participants were diagnosed by National Institute of Neurological and Communicative Disorders and Stroke-Alzheimer's disease and Related Disorders Association criteria [17] and Clinical Dementia Rating of 1.0-2.0, establishing mild to moderate $\mathrm{AD}$ [18]. Informed consent was obtained from subject or legal guardians in accord with the Institutional Review Board for Human Study at the University of Jordan/Faculty of Medicine. The AD patients had no major medical illnesses such as hypercholesterolemia, hypertension, renal disease, diabetes, etc., no psychiatric illnesses, and no systemic use of anti-hyperuricemic drugs, lipid lowering agents, psychotropic drugs, or substance abuse. Information about subjects was gathered from a questionnaire completed by all participants. The questionnaire covered socio-demographic characteristics, lifestyle, family, and medical history including chronic diseases and medications.

Initially, all subjects were screened with the (MMSE-A). A total 165 individual agreed to participate in the study; after the interview and the application of the AD diagnostic criteria, only 40 subjects were eligible for the mild to moderate AD group. In parallel, a group of 50 controls was randomly selected from different sectors of the same hospital (employee, caregivers, and visitors) and only 41 completed the study. They were functionally independent and cognitively healthy.

\section{Mental scales}

The Mini-Mental State Examination (MMSE) is a widely used screening instrument for assessing dementia in older adults. It tests five areas of cognitive functions: orientation, registration, attention/calculation, recall, and language [19]. MMSE was translated to the Arabic language and was adapted to the local Arabic dialogue [16]. Taking into consideration the high rate of illiteracy among the elderly population, the cutoff point for the presence of cognitive impairment was set according to the corresponding levels of education. For the control group. a score at 21/30 was set for those with no formal education, 23/30 for those who received $<12$ years of education, and 25/30 for those who had $>12$ 
years of education [16]. AD patients included in this study scored no less than 11 .

\section{Uric acid measurement}

Blood samples were centrifuged at 4,000 rpm for 2-3 min. Serum UA was measured with an invitro enzymatic colorimetric test [20] (UA Plus, Roche/Hitachi Diagnostics GmbH, Mannheim, Germany) then processed in the Cobas-Roche/Hitachi 902 analyzer at the University hospital laboratories.

\section{Lipid profile measurement}

The lipid profile measurements include serum total cholesterol (TC), triglycerides (TG), HDL, and LDL. All samples were measured by Roche Diagnostics COBAS INTEGRA 800 Biochemistry analyzer based on an enzymatic colorimetric method [21, 22].

\section{Statistical analysis}

Demographic, clinical, and laboratory data were coded and entered into SPSS for windows 20.0 (SPSS Inc., Chicago, IL). Data were summarized as means and standard deviations for continuous variables (age, UA, TC, TG, HDL, and LDL) or frequency and percentages for categorical variables (gender, education level, and smoking). The association between continuous variables and mental status was assessed by comparisons of means using $t$-test (control and AD groups). The association between categorical variables and mental status (control and AD groups) was examined utilizing Chi-square test and binary logistic regression. Bivariate correlations of the MMSE scores and the continuous variables was analyzed and presented as Pearson correlation coefficients. Statistical significance was set at $p<0.05$. Multivarient linear regression was used to measure the predictive value of continuous variables on MMSE scores.

\section{RESULTS}

\section{Demographic variables}

Forty AD patients and 41 control subjects completed the study. There are no significant difference between $\mathrm{AD}$ and control groups for age $(p=0.117)$ and gender $(p=0.888)$, respectively (Table 1$)$. Educational level shows significant difference between the $\mathrm{AD}$ and control groups, illiterates demonstrated a 7.5 fold $(p=0.033)$ increase in risk of developing AD.

\section{Mental scales}

Table 2 demonstrates the results of MMSE in the two groups. They are significantly different $(p=0.000)$.

\section{Biochemical parameters}

The means and standard deviations of the biochemical parameters in AD group and control subjects

Table 1

Demographics characteristics of study participants with AD versus control subjects

\begin{tabular}{|c|c|c|c|c|c|c|c|}
\hline Characteristic & Patients with AD & $n$ & Controls & $n$ & $p$-value & OR & $95 \% \mathrm{CI}$ \\
\hline Age* (Years) & $71.50 \pm 9.11$ & 40 & $68.46 \pm 8.13$ & 41 & 0.117 & & \\
\hline Gender $(\%)$ & & 40 & & 41 & 0.888 & & \\
\hline Male & $25(30.9 \%)$ & & $25(30.9 \%)$ & & & & \\
\hline Female & $15(18.5 \%)$ & & $16(19.8 \%)$ & & & & \\
\hline Educational Levels (\%) & & 40 & & 26 & 0.053 & & \\
\hline Illiterate & $21(52.5 \%)$ & & $7(26.9 \%)$ & & $0.033^{\dagger}$ & 7.50 & $1.18-47.68$ \\
\hline Less than 12 & $17(42.5 \%)$ & & $14(53.8 \%)$ & & 0.223 & 3.04 & $0.51-18.11$ \\
\hline More than 12 & $2(5 \%)$ & & $5(19.2 \%)$ & & 0.110 & 2.47 & $0.81-7.497$ \\
\hline Smoking (\%) & & 40 & & 26 & 0.105 & & \\
\hline Non smoker & $22(55 \%)$ & & $9(34.6 \%)$ & & & & \\
\hline Smokers & $18(45 \%)$ & & $17(65.4 \%)$ & & & & \\
\hline
\end{tabular}

OR, odds ratio; $95 \% \mathrm{CI}, 95 \%$ confidence interval. ${ }^{\dagger} p$-value is significant. ${ }^{*}$ Values shown $=$ mean \pm standard deviation (SD).

Table 2

MMSE results in both $\mathrm{AD}$ and control group

\begin{tabular}{lcccccc}
\hline Test & Patients with AD & $n$ & Control & $N$ & $p$-value & $95 \%$ CI \\
\hline MMSE* $^{2}$ & $14.90 \pm 5.57$ & 40 & $25.19 \pm 2.61$ & 41 & $0.000^{\dagger}$ & $-12.63--7.96$ \\
\hline
\end{tabular}

MMSE, Mini-Mental State Examination; 95\% CI, 95\% confidence interval. ${ }^{\dagger} p$-value is significant. ${ }^{*}$ Values shown $=$ mean \pm standard deviation $(\mathrm{SD})$. 
Table 3

Biochemical parameter values of individuals with AD versus controls

\begin{tabular}{lcccc}
\hline Biochemical test & Patient with AD & Control & $p$-value & $95 \%$ CI \\
\hline $\mathrm{UA}^{*}(\mathrm{mg} / \mathrm{dl})$ & $5.05 \pm 1.28$ & $5.78 \pm 1.70$ & $0.033^{\dagger}$ & $-1.39--0.059$ \\
$\mathrm{TC}^{*}(\mathrm{mg} / \mathrm{dl})$ & $184.24 \pm 42.39$ & $183.93 \pm 34.48$ & 0.976 & $0.987-1.01$ \\
$\mathrm{TG}^{*}(\mathrm{mg} / \mathrm{dl})$ & $163.34 \pm 65.14$ & $168.17 \pm 96.59$ & 0.114 & $-71.56-7.89$ \\
$\mathrm{HDL}^{*}(\mathrm{mg} / \mathrm{dl})$ & $41.95 \pm 9.21$ & $40.65 \pm 11.96$ & 0.620 & $-3.92-6.52$ \\
$\mathrm{LDL}^{*}(\mathrm{mg} / \mathrm{dl})$ & $118.36 \pm 34.44$ & $110.17 \pm 27.73$ & 0.313 & $-7.90-24.29$ \\
\hline
\end{tabular}

${ }^{\dagger} p$-value is significant. ${ }^{*}$ Values shown $=$ mean \pm standard deviation (SD). 95\% CI, 95\% confidence interval; UA, uric acid;

TC, total cholesterol; TG, triglycerides; HDL, high density lipoprotein; LDL, low density lipoprotein.

are shown in Table 3. Serum UA is lower in the $\mathrm{AD}$ group $(5.05 \pm 1.28)$ compared to control group $(5.78 \pm 1.70)$. The difference between the two groups is small numerically, however it is statistically significant $(p=0.033)$ and the values are within the normal physiological reference range (Serum UA levels ranges from $1.5-7.1 \mathrm{mg} / \mathrm{dL}$ for women and $1.5-7.6 \mathrm{mg} / \mathrm{dL}$ for men).

The lipid profile analysis in the two groups shows slightly higher than normal TG and LDL and lower HDL when compared to the National Cholesterol Education Program/healthy lipid profile guidelines ( TC $<200 \mathrm{mg} / \mathrm{dl}, \mathrm{TG}<150 \mathrm{mg} / \mathrm{dl}, \mathrm{HDL}>60 \mathrm{mg} / \mathrm{dl}$, and $\mathrm{LDL}<100 \mathrm{mg} / \mathrm{dl}$ ) [23]. There is no difference in lipid profile between the AD and control groups.

\section{Correlations and analysis}

Pearson Correlation and linear regression analysis do not show a correlation between MMSE scores and
UA, TC, TG, HDL, and LDL in AD group except for age $(p=0.020)$ (Table 4$)$ and $(p=0.006)$ (Table 5).

\section{DISCUSSION}

In this study, persons affected by AD are older and in general have received less years of formal education compared to the control group. Educational level has been proposed to correlate inversely with the risk of dementia [24]. For some, this finding is attributed to compensatory strategies adapted by educated individuals that may delay the detection of the disease, or it may be that people with greater cognitive reserve usually choose mentally stimulating leisure activities and jobs, leading to a chicken-and-egg dilemma for the interpretation of the relationship between mentally stimulating activities in adulthood and dementia risk [25].

Among possible markers of age-related cognitive decline, UA is controversial because it has antioxidant

Table 4

Correlation between MMSE and continuous variables for AD patients

\begin{tabular}{lcccccccc}
\hline & MMSE & Age & UA & TC & TG & HDL & LDL \\
\hline MMSE & Pearson correlation & 1 & $-0.367 *$ & -0.003 & -0.014 & 0.181 & -0.245 & -0.016 \\
& Sig. (2-tailed) & & $0.020^{*}$ & 0.986 & 0.933 & 0.264 & 0.127 \\
& $\mathrm{~N}$ & 40 & 40 & 40 & 40 & 40 & 40 & 40.921 \\
& & & & 40 & & 40 \\
\hline
\end{tabular}

*Correlation is significant at the 0.05 level (2-tailed). UA, uric acid; TC, total cholesterol; TG, triglycerides; HDL, high density lipoprotein; LDL, low density lipoprotein.

Table 5

Linear regression between MMSE and continuous variables for AD patients

\begin{tabular}{|c|c|c|c|c|c|c|c|}
\hline \multicolumn{8}{|c|}{ Coefficients $^{\mathrm{a}}$} \\
\hline \multirow[t]{2}{*}{ Model } & \multicolumn{2}{|c|}{$\begin{array}{l}\text { Unstandardized } \\
\text { coefficients }\end{array}$} & \multirow{2}{*}{$\begin{array}{c}\text { Standardized } \\
\text { coefficients } \\
\text { Beta }\end{array}$} & \multirow[t]{2}{*}{$\mathrm{t}$} & \multirow[t]{2}{*}{ Sig. } & \multicolumn{2}{|c|}{$95 \% \mathrm{CI}$ for $\mathrm{B}$} \\
\hline & B & Std. Error & & & & Lower Bound & Upper Bound \\
\hline (Constant) & 42.058 & 9.805 & & 4.289 & 0.000 & 22.109 & 62.006 \\
\hline Age & -0.281 & 0.096 & -0.459 & -2.920 & $0.006^{*}$ & -0.476 & -0.085 \\
\hline UA & -0.183 & 0.665 & -0.042 & -0.275 & 0.785 & -1.536 & 1.171 \\
\hline $\mathrm{TC}$ & 0.013 & 0.054 & 0.097 & 0.238 & 0.814 & -0.096 & 0.122 \\
\hline TG & 0.013 & 0.016 & 0.156 & 0.826 & 0.415 & -0.019 & 0.046 \\
\hline HDL & -0.176 & 0.104 & -0.290 & -1.682 & 0.102 & -0.388 & 0.037 \\
\hline LDL & -0.025 & 0.065 & -0.155 & -0.385 & 0.703 & -0.158 & 0.108 \\
\hline
\end{tabular}

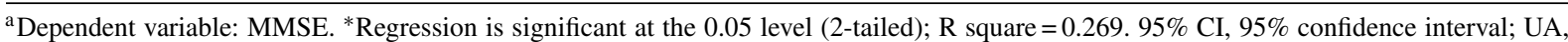
uric acid; TC, total cholesterol; TG, triglycerides; HDL, high density lipoprotein; LDL, low density lipoprotein. 
properties; in fact it accounts for up to $60 \%$ of the free radical scavenging activity in human blood. On the other hand, UA is increased in diseases that often lead to cognitive impairment. The mechanism linking UA to $\mathrm{AD}$ is unknown. In the present study, numerically small but statistically significant reduction in serum UA is demonstrated in AD patients as compared to those of the healthy controls. UA can scavenge superoxide and remove it by preventing superoxide dismutase enzyme degradation; this enzyme is responsible for clearing superoxide from the cell [26-28]. Removal of superoxide helps to prevent its reaction with nitric oxide and block the formation of peroxynitrite [29]. UA can prevent the inactivation of cellular enzymes by preventing peroxynitrite from nitrating the tyrosine residues of proteins, thus preventing modification of cytoskeleton [28]. UA can also prevent oxidative damage by its ability to bind to iron and inhibit iron-dependent ascorbate oxidation thus decrease production of free radicals [27]. Thus, a reduced UA concentration may decrease the ability of the body to prevent peroxynitrite and other free radicals from acting on cellular components and damaging the cell. Lower serum UA levels in $\mathrm{AD}$ as compared to control subjects in this study support the hypothesis that oxidative injuries may have an important role in the pathogenesis of $\mathrm{AD}$ and it is in line with previous studies $[3,30]$. There is an abundance of evidence that suggests that low UA levels are associated with the development and progression of a variety of diseases like multiple sclerosis and $\mathrm{AD}[13,31-33]$. Whether low serum UA levels are a cause or a consequence of these neurodegenerative diseases needs to be investigated. It is possible that persons with low serum UA levels are unable to prevent against free radical toxicity, leading to the development of inflammation and the destruction of tissues. However, it is also possible that the inflammation that occurs leads to the consumption of UA to scavenge the excess free radicals produced, resulting in a lower UA level [34]. The results of the direct administration of UA and subsequent increase in serum UA concentration in mice model of multiple sclerosis (experimental allergic encephalomyelitis) provide support that low UA levels are a cause, and not a consequence of the neuro-degenerative diseases at least in multiple sclerosis [35].

There is conflicting results in the literature concerning the relationship between TC and AD. These results may be explained by the timing of the TC measurements in relationship to age and the clinical onset of cognitive decline [36]. In our study, there is no difference in lipid profile between the AD and control groups: the AD group demonstrated a similar AD lipid profile pattern reported in previous study with the only exception being the TC levels $<200 \mathrm{mg} / \mathrm{dl}$ [37].

Pearson correlation and linear regression analysis do not show a correlation between MMSE and continuous variables (age, TC, TG, HDL, LDL, and UA) in the AD group except for age (Tables 4 and 5) indicating that UA and lipid profile cannot be used as biomarkers for early diagnosis of $\mathrm{AD}$ cases. $\mathrm{AD}$ is a disease of aging. Importantly, this holds true even in individuals with a genetic predisposition, i.e., those individuals with an autosomal dominant inheritance of $\mathrm{AD}$ or in individuals with Down's syndrome who develop the pathology of AD. Therefore age is a clear contributor in $100 \%$ of AD cases, whatever the genetic background. The aging process is associated with an increase in the adventitious production of oxygen-derived radicals and also a decrease in the ability to defend against such radicals [3]. Not surprisingly, studies over the past 10 years have established oxidative stress and damage not only in the lesions of AD but also in neurons at risk of death [38-41].

The results presented here may imply the presence of a more integral patho-physiological mechanism for AD. Genetic studies, neuroimaging techniques, and research for biological markers in general can help clarify the pathophysiology of $\mathrm{AD}$ leading to more effective therapeutic strategies.

\section{Limitations}

The present study is a cross-sectional analysis involving only one clinical and laboratory evaluation of the serum UA and lipid profile of the individuals without any further follow-up. The changes in UA, TC, TG, HDL, and LDL levels over time could not be taken into consideration, and therefore, it is not possible to discriminate whether there were differences in serum UA and lipid levels that preceded the onset of the dementia or whether they developed during the course of the diseases.

\section{Conclusion}

Serum UA is significantly lower in the AD patients than in the healthy controls. This study supports the hypothesis that oxidative injuries could play an important role in the pathogenesis of AD. Further studies to investigate the association of serum UA with clinical course and treatment response in AD will need to be conducted. Lipid profile in the two groups is statistically not significant. Linear regression analysis do 
not establish serum UA or lipid profile as a potential predictor to cognitive impairment; however, it does enhance the necessity for further investigation, possibly within the framework of a common underlying pathophysiology for dementia in the elderly.

\section{ACKNOWLEDGMENTS}

This work was supported by Grant 1135 from the Deanship of Scientific Research of the University of Jordan.

Authors' disclosures available online (http://www.jalz.com/disclosures/view.php?id=2573).

\section{REFERENCES}

[1] Al-Makhamreh S, Hasna F, Al-Khateeb E (2011) The forgotten few: The social context of ageing and Alzheimer's disease in Jordan. British Society of Gerontology's Generations Review, http://www.britishgerontology.org/DB/gr-editions2/generations-review/the-forgotten-few-the-social-contextof-ageing-a-2.html.

[2] Christen Y (2000) Oxidative stress and Alzheimer disease. Am J Clin Nutr 71, 621-629.

[3] Zhu X, Raina AK, Perry G, Smith MA (2004) Alzheimer's disease: The two-hit hypothesis. Lancet Neurol 3, 219-226.

[4] Smith MA, Nunomura A, Zhu X, Takeda A, Perry G (2000) Metabolic, metallic, and mitotic sources of oxidative stress in AD. Redox Signal 2, 413-420.

[5] Sparks DL, Petanceska S, Sabbagh M, Connor D, Soares H, Adler C, Lopez J, Ziolkowski C, Lochhead J, Browne P (2005) Cholesterol, copper and Abeta in controls, MCI, AD and the AD cholesterol-lowering treatment trial (ADCLT). Curr Alzheimer Res 2, 495-496.

[6] Tan Z, Seshadri S, Beiser A, Wilson PW, Kiel DP, Tocco M, D'Agostino RB, Wolf PA (2003) Plasma total cholesterol level as a risk factor for Alzheimer disease: The Framingham Study. Arch Intern Med 163, 1053-1057.

[7] Caramelli P, Nitrini R, Maranhao R, Lourenço ACG, Damasceno MC, Vinagre C, Caramelli B (1999) Increase apolipoprotin B serum concentration in Alzheimer's disease. Acta Neurol Scand 100, 61-63.

[8] Fang J, Alderman MH (2000) Serum uric acid and cardiovascular mortality; The NHANES I epidemiologic follow-up study, 1971-1992. JAMA 283, 2404-2410.

[9] Bos MJ, Koudstaal PJ, Hofman A, Witteman JCM, Breteler MMB (2006) Uric acid is a risk factor for myocardial infarction and stroke; The Rotterdam Study. Stroke 37, 1503-1510.

[10] Johnson RJ, Kang DH, Feig D, Kivlighn S, Kanellis J, Watanabe S, Rodriguez-Iturbe R, Mazzali M (2003) Is there a pathogenetic role for uric acid in hypertension and cardiovascular and renal disease? Hypertension 41, 1183-1190.

[11] Miller NJ, Rice-Evans C, Davies MJ, Gopinathan V, Milner A (1993) A novel method for measuring antioxidant capacity and its application to monitoring the antioxidant status in premature neonates. Clin Sci 84, 407-412.

[12] Rinaldi P, Polidori MC, Metastasio A, Mariani E, Mattioli P, Cherubini A, Catani M, Cecchetti R, Senin U, Mecocci P (2003) Plasma antioxidants are similarly depleted in mild cognitive impairment and in Alzheimer's disease. Neurobiol Aging 24, 915-919.
[13] Kim TS, Pae CU, Yoon SJ, Jang WY, Lee NJ, Kim JJ, Lee SJ, Lee C, Paik IH, Lee CU (2006) Decreased plasma antioxidants in patients with Alzheimer's disease. Int J Geriatr Psychiatry 21, 344-348.

[14] Irizarry MC, Raman R, Schwarzschild MA, Becerra LM, Thomas RG, Peterson RC, Ascherio A, Aisen PS (2009) Plasma urate and progression of mild cognitive impairment. Neurodegener Dis 6, 23-28.

[15] Polidori MC, Mecocci P (2002) Plasma susceptibility to free radical-induced antioxidant consumption and lipid peroxidation is increased in very old subjects with Alzheimer disease. J Alzheimers Dis 4, 517-522.

[16] Al-khateeb E, Al-zayadneh E, Al-dalahmah O, Alawadi Z, Khatib F, Naffa R, Shafagoj Y (2014) Relation between copper, lipid profile, and cognition in elderly Jordanians. J Alzheimers Dis 41, 203-211.

[17] McKhann G, Drachman D, Folstein M, Katzman R, Price D, Stadlan EM (1984) Clinical diagnosis of AD; report of the NINCDS-ADRDA work group under the auspices of Department of Health and Human Services Task Force on AD. Neurology 34, 939-944.

[18] Morris JC (1993) The Clinical Dementia Rating (CDR): Current version and scoring rules. Neurology 43, 2412-2414.

[19] Folstein MF, Folstein SE, McHugh PR (1975) Mini Mental State. A practical method for grading the cognitive state of patients for clinician. J Psychiatr Res 12, 189-198.

[20] Ruiz García A, Sánchez Armengol A, LuqueCrespo E, García Aguilar D, Romero Falcón A, Carmona Bernal C, Capote F (2006) Blood uric acid levels in patients with sleep-disordered breathing. Arch Bronconeumol 42, 492-500.

[21] Siedel J, Hagele EO, Ziegenhorn J, Wahiefeld AW (1983) Reagent for the enzymatic determination of serum total cholesterol with improved lipolytic efficiency. Clin. Chem 29, 1075-1080.

[22] Lopes-Virella MF, Stone P, Ellis S, Colwell JA (1977) Cholesterol determination in high-density lipoproteins separation by three different methods. Clin Chem 23, 882-884.

[23] National Cholesterol Education Program: Third report of the National Cholesterol Education Program (NCEP) expert panel on detection, evaluation and treatment of high blood cholesterol in adults (adult treatment panel III). NIH Publication 01-3670, (2001).

[24] Stern Y, Alexander GE, Prohovnik I, Mayeux R (1992) Inverse relationship between education \&parietotemporal perfusion deficit in AD. Ann Neurol 32, 371-375.

[25] Gatz M (2005) Educating the brain to avoid dementia: Can mental exercise prevent Alzheimer disease? PLoS Med 2, e7.

[26] Ames BN, Cathcart R, Schwiers E, Hochstein P (1981) Uric acid provides an antioxidant defense in humans against oxidant- and radical-caused aging and cancer: A hypothesis. Proc Natl Acad Sci U S A 78, 6858-6862.

[27] Davies KJ, Sevanian A, Muakkassah-Kelly SF, Hochstein P (1986) Uric acid iron ion complexes. A new aspect of the antioxidant functions of uric acid. Biochem $J \mathbf{2 3 5}$, 747-754

[28] Pacher P, Beckman JS, Liaudet L (2007) Nitric oxide and peroxynitrite in health and disease. Physiol Rev 87, 315-424.

[29] Van der Veen RC, Hinton DR, Incardonna F, Hofman FM (1997) Extensive peroxynitrite activity during progressive stages of central nervous system inflammation. J Neuroimmunol 77, 1-7.

[30] Zaman Z, Roche S, Fielden P, Frost PG, Niriella DC, Cayley AC (1992) plasma concentrations of vitamins A and E and carotenoids in Alzheimer's disease. Age Ageing 21, 91-94. 
[31] Spitsin S, Hooper DC, Mikheeva T, Koprowski H (2001) Uric acid levels in patients with multiple sclerosis: Analysis in mono- and dizygotic twins. Mult Scler 7, 165-166.

[32] Toncev G, Milicic B, Toncev S, Samardzic G (2002) Serum uric acid levels in multiple sclerosis patients correlate with activity of disease and blood-brain barrier dysfunction. Eur J Neurol 9, 221-226.

[33] Rentzos M, Nikolaou C, Anagnostouli M, Rombos A, Tsakanikas K, Economou M, Dimitrakopoulos A, Karouli M, Vassilopoulos D (2006) Serum uric acid and multiple sclerosis. Clin Neurol Neurosurg 108, 527-531.

[34] Drulovic J, Dujmovic I, Stojsavljevic N, Mesaros S, Andjelkovic S, Miljkovic D, Peric V, Dragutinovic G, Marinkovic J, Levic Z, Stojkovic MM (2001) Uric acid levels in sera from patients with multiple sclerosis. J Neurol 248, 121-126.

[35] Hooper DC, Scott GS, Zborek A, Mikheeva T, Kean RB, Koprowski H, Spitsin SV (2000) Uric acid, a peroxynitrite scavenger, inhibits CNS inflammation, blood-CNS barrier permeability changes, and tissue damage in a mouse model of multiple sclerosis. FASEB J 14, 691-698.

[36] Hofman A, Ott A, Breteler MM, Bots ML, Slooter AJ, van Harskamp $\mathrm{F}$, van Duijn $\mathrm{CN}$, Van Broeckhoven $\mathrm{C}$,
Grobbee DE (1997) Atherosclerosis, apolipoprotein E, and prevalence of dementia and Alzheimer's disease in the Rotterdam study. Lancet 349, 151-154.

[37] Sabbagh M, Zahiri HR, Ceimo J, Cooper K, Gaul W, Connor D, Sparks DL (2004) Is there a characteristic lipid profile in Alzheimer's disease? J Alzheimers Dis 6, 585-589.

[38] Smith MA, Richey PL, Taneda S., Kutty RK, Sayre LM, Monnier VM, Perry G (1994) Advanced Maillard reaction end products, free radicals, and protein oxidation in Alzheimer's disease, Ann N Y Acad Sci 738, 447-454.

[39] Smith MA, Sayre LM, Monnier VM, Perry G (1995) Radical ageing in Alzheimer's disease. Trends Neurosci 18, 172-176.

[40] Smith MA, Perry G, Richey PL, Sayre LM, Anderson VE, Beal MF, Kowall N (1996) Oxidative damage in Alzheimer's. Nature 382, 120-121.

[41] Smith MA, Richey PL, Harris LM, Sayre LM, Beckman JS, Perry G (1997) Widespread peroxynitrite-mediated damage in Alzheimer's disease. J Neurosci 17 2653-2657. 